\title{
POINTWISE ESTIMATE FOR THE BERGMAN KERNEL OF THE WEIGHTED BERGMAN SPACES WITH EXPONENTIAL TYPE WEIGHTS
}

\author{
SAÏD ASSERDA AND AMAL HICHAME
}

\begin{abstract}
Let $A L_{\phi}^{2}(\mathbb{D})$ denote the closed subspace of $L^{2}\left(\mathbb{D}, e^{-2 \phi} d \lambda\right)$ consisting of holomorphic functions in the unit disc $\mathbb{D}$. For certain class of subharmonic funcions $\phi: \mathbb{D} \rightarrow \mathbb{D}$, we prove upper pointwise estimate for the Bergman kernel for $A L_{\phi}^{2}(\mathbb{D})$.
\end{abstract}

\section{INTRODUCTION AND STATEMENT OF MAIN RESUlT}

Let $\mathbb{D}$ be the unit disc in $\mathbb{C}$ and $d \lambda$ be its Lebesgue measure. For a measurable function $\phi: \mathbb{D} \rightarrow \mathbb{D}$, let $L_{\phi}^{2}(\mathbb{D})$ be the Hilbert space of measurable function $f$ on $\mathbb{D}$ such that

$$
\|f\|_{L_{\phi}^{2}}:=\left(\int_{\mathbb{D}}|f|^{2} e^{-2 \phi} d \lambda\right)^{\frac{1}{2}}<\infty
$$

Let $A L_{\phi}^{2}(\mathbb{D})$ be the closed subspace of $L_{\phi}^{2}(\mathbb{D})$ consisting of analytic functions. Let $P$ be the orthogonal projection of $L_{\phi}^{2}(\mathbb{D})$ onto $A L_{\phi}^{2}(\mathbb{D})$ :

$$
P f(z):=\int_{\mathbb{D}} K(z, w) f(w) e^{-2 \phi(w)} d \lambda
$$

where $K$ is the reproducing kernel of $P$.

The purpose of this note is to give an upper pointwise estimate of $K$ for some class of subharmonic functions $\phi$ on $\mathbb{D}$ introduced by Oleinik [10] and OleinikPerel'man [11].

Definition 1.1. For $\phi \in C^{2}(\mathbb{D})$ and $\Delta \phi>0$ put $\tau=(\Delta \phi)^{-1 / 2}$ where $\Delta$ is the Laplace operator. We call $\phi \in \mathcal{O P}(\mathbb{D})$ if the following conditions holds.

(1) $\exists C_{1}>0$ such that $|\tau(z)-\tau(w)| \leq C_{1}|z-w|$,

(2) $\exists C_{2}>0$ such that $\tau(z) \leq C_{2}(1-|z|)$,

(3) $\exists 0<C_{3}<1$ and $a>0$ such that $\tau(w) \leq \tau(z)+C_{3}|z-w|$ for $w \notin D(z, a \tau(z))$ where $D(z, a \tau(z))=\{w \in \mathbb{D},|w-z| \leq a \tau(z)\}$.

Some examples of functions in $\mathcal{O P}(\mathbb{D})$ are as follows :

(i) $\phi_{1}(z)=-\frac{A}{2} \log \left(1-|z|^{2}\right), \quad A>0$.

(ii) $\phi_{2}(z)=\frac{1}{2}\left(-A \log \left(1-|z|^{2}\right)+B\left(1-|z|^{2}\right)^{-\alpha}\right), A \geq 0, B>0, \alpha>0$.

(iii) $\phi_{1}+h$ and $\phi_{2}+h$ where $\phi_{1}$ and $\phi_{2}$ are as in (i) and (ii) respectively and

Date: October 7, 2018.

2010 Mathematics Subject Classification. Primary 32A25, Secondary 30H20.

Key words and phrases. Bergman Kernel, $\bar{\partial}$-equation. 
$h \in C^{2}(\mathbb{D})$ can be any harmonic function on $\mathbb{D}$.

For $z, w \in \mathbb{D}$, the distance $d_{\phi}$ induced by the metric $\tau(z)^{-2} d z \otimes d \bar{z}$ is given by

$$
d_{\phi}(z, w)=\inf _{\gamma} \int_{0}^{1} \frac{\left|\gamma^{\prime}(t)\right|}{\tau(\gamma(t))} d t
$$

where $\gamma$ runs over the piecewise $C^{1}$ curves $\gamma:[0,1] \rightarrow \mathbb{D}$ with $\gamma(0)=z$ and $\gamma(1)=w$. Thanks to condition (2) the metric space $\left(\mathbb{D}, d_{\phi}\right)$ is complete and $d_{\phi} \succeq d_{h}$ where $d_{h}$ is the hyperbolic distance.

Our main result is the following theorem on the off-diagonal decay of the Bergman kernel.

Theorem 1.2. Let $\phi \in \mathcal{O P}(\mathbb{D})$ and $K$ be the Bergman kernel for $A L_{\phi}^{2}(\mathbb{D})$. There exist positive constants $C$ and $\sigma$ such that for any $z, w \in \mathbb{D}$

$$
|K(z, w)| e^{-(\phi(z)+\phi(w))} \leq C \frac{1}{\tau(z) \tau(w)} \exp \left(-\sigma d_{\phi}(z, w)\right)
$$

In [4] and [9] M.Christ and J.Marzo-J.Ortega-Cerdà obtained a pointwise estimates for the Bergman kernel of the weighted Fock space $\mathcal{F}_{\phi}^{2}(\mathbb{C})$ under the hypothesis that $\Delta \phi$ is a doubling measure. This result was extended to several variables by H.Delin and H.Lindholm in [5] and [7] under similar hypothesis. In [12], A.P.Schuster and D.Varolin obtained a pointwise estimate for the Bergman Kernel of the weighted Bergman space $A L^{2}\left(\mathbb{D}, e^{-2 \phi}\left(1-|z|^{2}\right)^{-2} d \lambda\right)$ under the hypothesis that $\Delta \phi$ is comparable to hyperbolic metric of $\mathbb{D}$ :

$$
|K(z, w)| e^{-(\phi(z)+\phi(w))} \leq C \exp \left(-\sigma d_{h}(z, w)\right)
$$

For $\phi \in \mathcal{O P}(\mathbb{D})$ and under the strong condition : $\forall m \geq 1, \exists b_{m}>0$ and $0<t_{m}<\frac{1}{m}$ such that

$$
\tau(w) \leq \tau(z)+t_{m}|z-w| \text { if }|z-w|>b_{m} \tau(z),
$$

H.Arroussi and J.Pau [1] give the following pointwise estimate : for each $k \geq 1$ there exists $C_{k}>0$ such that

$$
|K(z, w)| e^{-(\phi(z)+\phi(w))} \leq \frac{C_{k}\left[d_{\tau}(z, w)\right]^{-k}}{\tau(z) \tau(w)}
$$

where $d_{\tau}(w)=\frac{|z-w|}{\min [\tau(z), \tau(w)]}$. A better estimate will be

$$
|K(z, w)| e^{-(\phi(z)+\phi(w))} \leq \frac{C}{\tau(z) \tau(w)} e^{-\sigma d_{\tau}(z, w)} .
$$

\section{Proof of theorem 1.2}

Near the diagonal, by [8,lemma 3.6 ] there exists $\alpha>0$ sufficiently small such that

$$
|K(z, w)| \sim \sqrt{K(z, z)} \sqrt{K(w, w)} \sim \frac{e^{\phi(z)+\phi(w)}}{\tau(z) \tau(w)} \quad \text { if } \quad|z-w| \leq \alpha \min [\tau(z), \tau(w)]
$$

Off the diagonal, let $|z-w|>\alpha \min [\tau(z), \tau(w)]$ and $\beta>0$ such that $D(z, \beta \tau(z)) \cap$ $D(w, \beta \tau(w))=\emptyset$. We may suppose that $\tau(z) \leq \tau(w)$. Fix a smooth function $\chi \in C_{0}^{\infty}(\mathbb{D})$ such that 
$-\operatorname{supp} \chi \subset D(w, \beta \tau(w))$,

$-0 \leq \chi \leq 1, \chi=1$ in $D\left(w, \frac{\beta}{2} \tau(w)\right)$ and

$-|\bar{\partial} \chi|^{2} \preceq \chi \tau(w)^{-2}$.

Since $\phi \in \mathcal{O P}(\mathbb{D})$, by [10,lemma 1 and 2] the following mean inequality holds

$$
\begin{aligned}
\mid K(w, z))\left.\right|^{2} e^{-2 \phi(w)} & \left.\preceq \frac{1}{\tau(w)^{2}} \int_{D\left(w, \frac{\beta}{2} \tau(w)\right)} \chi(\zeta) \mid K(\zeta, z)\right)\left.\right|^{2} e^{-2 \phi(\zeta)} d \lambda(\zeta) \\
& \preceq \frac{1}{\tau(w)^{2}}\|K(., z)\|_{L^{2}\left(\chi e^{-2 \phi} d \lambda\right)}^{2}
\end{aligned}
$$

Hence

$$
\|K(., z)\|_{L^{2}\left(\chi e^{-\phi}\right)}=\sup _{f}\left|<f, K(., z)>_{L^{2}\left(\chi e^{-2 \phi} d \lambda\right)}\right|
$$

where $f$ is holomorphic in $D(w, \beta \tau(w))$ with $\|f\|_{L^{2}\left(\chi e^{-2 \phi} d \lambda\right)}=1$. Since $P_{\phi}(f \chi)(z)=<$ $f, K(., z)>_{L^{2}\left(\chi e^{-2 \phi} d \lambda\right)}$ and that $u_{f}=f \chi-P_{\phi}(f \chi)$ is the minimal solution in $L^{2}\left(\mathbb{D}, e^{-2 \phi} d \lambda\right)$ of $\bar{\partial} u=f \bar{\partial} \chi$, and from the fact $\chi(z)=0$, we have

$$
\left|<f, K(., z)>_{L^{2}\left(\chi e^{-2 \phi} d \lambda\right)}\right|=\left|P_{\phi}(f \chi)(z)\right|=\left|u_{f}(z)\right|
$$

Since $D(z, \beta \tau(z)) \cap D(w, \beta \tau(w))=\emptyset$, the function $u_{f}$ is holomorphic in $D(z, \nu \tau(z))$ for some $\nu>0$. By the mean value inequality

$$
\begin{aligned}
\left|u_{f}(z)\right|^{2} e^{-2 \phi(z)} & \preceq \frac{1}{\tau(z)^{2}} \int_{D(z, \nu \tau(z))}\left|u_{f}(\zeta)\right|^{2} e^{-2 \phi(\zeta)} d \lambda \\
& \preceq \frac{1}{\tau(z)^{2}} \int_{D(z, \nu \tau(z))} e^{-\epsilon \frac{|\zeta-z|}{\nu \tau(z)} \mid}\left|u_{f}(\zeta)\right|^{2} e^{-2 \phi(\zeta)} d \lambda
\end{aligned}
$$

Since the linear curve $\gamma(t)=(1-t) z+t \zeta$ lies in $D(z, \nu \tau(z))$ and $\tau(\gamma(t)) \sim \tau(z)$, we have $d_{\phi}(\zeta, z) \leq C \frac{|\zeta-z|}{\tau(z)}$ for $\zeta \in D(z, \nu \tau(z))$. Hence

$$
\begin{aligned}
\left|u_{f}(z)\right|^{2} e^{-2 \phi(z)} & \preceq \frac{1}{\tau(z)^{2}} \int_{D(z, \nu \tau(z))} e^{-C \epsilon d_{\phi}(\zeta, z)}\left|u_{f}(\zeta)\right|^{2} e^{-2 \phi(\zeta)} d \lambda \\
& \preceq \frac{1}{\tau(z)^{2}} \int_{\mathbb{D}} e^{-C \epsilon d_{\phi}(\zeta, z)}\left|u_{f}(\zeta)\right|^{2} e^{-2 \phi(\zeta)} d \lambda
\end{aligned}
$$

The function $\zeta \rightarrow d_{\phi}(\zeta, z)$ is smooth on $\mathbb{D} \backslash \operatorname{Cut}(z) \cup\{z\}$ where $\operatorname{Cut}(z)$ is the cut locus : the set of all cut points of $z$ along all geodesics that start from $z$. To get a smooth Lipschitz approximation of $d_{\phi}$, we recall the following result of Greene-Wu [6] ( see also [2]).

Theorem 2.1. Let $M$ be a complete Riemannian manifold, let $h: M \rightarrow \mathbb{R}$ be a Lipschitz function, let $\eta: M \rightarrow] 0,+\infty$ [ be a continuous function, and $r$ a positive number. Then there exist a smooth Lipschitz function $g: M \rightarrow \mathbb{R}$ such that $|h(x)-g(x)| \leq \eta(x)$ for every $x \in M$, and $\operatorname{Lip}(g) \leq \operatorname{Lip}(h)+r$.

We use this result with $h(\zeta)=d_{\phi}(\zeta, z), \eta=1$ and $r=1$. We have $d_{\phi}(\zeta, z) \prec$ $g_{z}(\zeta) \prec d_{\phi}(\zeta, z)$ and $\tau(\zeta)\left|d g_{z}(\zeta)\right| \leq 2$. Hence

$$
\left|u_{f}(z)\right|^{2} e^{-2 \phi(z)} \preceq \frac{1}{\tau(z)^{2}} \int_{\mathbb{D}} e^{-C \epsilon g_{z}(\zeta)}\left|u_{f}(\zeta)\right|^{2} e^{-2 \phi(\zeta)} d \lambda
$$


By Berndtsson-Delin's improved $L^{2}$ estimates of for the minimal solution of $\bar{\partial}$ in $L^{2}\left(\mathbb{D}, e^{-2 \phi} d \lambda\right)[3][5]$, we have :

$$
\int_{\mathbb{D}} e^{-C \epsilon g_{z}(\zeta)}\left|u_{f}(\zeta)\right|^{2} e^{-2 \phi(\zeta)} d \lambda \preceq \int_{\mathbb{D}} e^{-C \epsilon g_{z}(\zeta)}|\bar{\partial} \chi(\zeta)|^{2}|f(\zeta)|^{2} \tau(\zeta)^{2} e^{-2 \phi(\zeta)} d \lambda
$$

provided that $\tau\left|\partial \omega_{\epsilon}\right| \leq \mu \omega_{\epsilon}$ with $\mu<\sqrt{2}$ where $\omega_{\epsilon}(\zeta)=e^{-C \epsilon g_{z}(\zeta)}$. If we choose $\epsilon$ small enough so that $\mu=2 C \epsilon<\sqrt{2}$ then $\tau\left|\partial \omega_{\epsilon}\right|=C \epsilon \tau\left|\partial g_{z}\right| \omega_{\epsilon} \leq \mu \omega_{\epsilon}$. Thus

$$
\left|u_{f}(z)\right|^{2} e^{-2 \phi(z)} \preceq \frac{1}{\tau(z)^{2}} \int_{D(w, \beta \tau(w))} e^{-C \epsilon d_{\phi}(\zeta, z)} \chi(\zeta)|f|^{2} e^{-2 \phi(\zeta)} d \lambda
$$

where for the last term we use $\tau(\zeta) \sim \tau(w)$. Since $\zeta \in D(w, \beta \tau(w))$ we have

$$
d_{\phi}(\zeta, z) \geq d_{\phi}(z, w)-d_{\phi}(w, \zeta) \succeq d_{\phi}(z, w)-\frac{|\zeta-w|}{\beta \tau(w)} \succeq d_{\phi}(z, w)
$$

and thanks to $(*)$, we conclude

$$
|K(z, w)| e^{-(\phi(w)+\phi(z))} \leq \frac{C}{\tau(z) \tau(w)} e^{-\sigma d_{\phi}(z, w)} .
$$

\section{REFERENCES}

[1] H.Arroussi and J.Pau, Reproducing kernel estimates, bounded projections and duality on large weighted Bergman spaces. arXiv:1309.6072v1[math.CV]24 Sep 2013.

[2] D.Azagra,J.Ferrera,F.Lopez-Mesas and Y.Rangel, Smooth approximation of Lipschitz functions on Riemannian manifolds. J.Math.Anal.Appl. 326, 1370-1378, 2007.

[3] B.Berndtsson, Weighted estimates for the $\bar{\partial}$-equation. Complex analysis and geometry (Columbus OH,1999), 43-57, Ohio State Univ.Math.Res.Inst.Publ. 9, De Gruyter Berlin, 2001.

[4] M.Christ, On the $\bar{\partial}$-equation in weighted $L^{2}$ norm in $\mathbb{C}$, J.Geom.Anal. 1, no 3, 193-230, 1991.

[5] H.Delin, Pointwise estimates for the weighted Bergman projection kernel in $\mathbb{C}^{n}$ using a weighted $L^{2}$ estimate for the $\bar{\partial}$ equation, Ann.Inst.Fourier(Grenoble) 48, no 4, 967-997, 1998.

[6] R.E.Greene and H.Wu, $C^{\infty}$ approximations of convex, subharmonic, and plurisubhrmonic functions. Ann.Sci.École Norm.Sup. (4) 12 (1), 47-84, 1979.

[7] N.Lindholm, Sampling in weighted $L^{p}$ spaces of entire function in $\mathbb{C}^{n}$ and estimates of the Bergman kernel, J.Funct.Anal. 182, 390-426, 2001.

[8] P.Lin and R.Rochberg, Trace ideal criteria for Toeplitz and Hankel operators on the weighted Bergman spaces with exponential type weights, Pacific.J.Math. 173, no 1, 127$146,1996$.

[9] J.Marzo and J.Ortega-Cerdà Pointwise estimates for the Bergman kernel of the weighted Fock space, J.Geom.Anal. 19, 890-910 2009.

[10] V.L.Oleinik and B.S.Pavlov Imbedding theorems for weighted classes of harmonic and analytic functions, J. Soviet Math. 2, 135-142, 1974, translation in: Zap. Nauchn. Sem. LOMI Steklov 22, 1971.

[11] V.L.Oleinik and G.S.Perel'man, Carleson's Imbedding theorems for a weighted Bergman spaces, Math.Notes, 7, 577-581, 1990.

[12] A.P.Schuster and D.Varolin New estimates for the minimal $L^{2}$ solution of $\bar{\partial}$ and application to geometric function theory in weighted Bergman spaces, J.Rei.Angew.Math. De Gruyter, DOI: $10.1515 /$ crelle-2012-0072. 
POINTWISE ESTIMATE FOR THE BERGMAN KERNEL

IBN TOFAIL UNIVERSITY, FACULTy OF SCIENCES, DEPARTMENT OF MATHEMATICS, PO 242 Kenitra Morroco

E-mail address: asserda-said@univ-ibntofail.ac.ma

Regional Centre of trades of education and training, Kenitra Morocco

E-mail address: amalhichame@yahoo.fr 